$\Rightarrow$ CELL ADHESION

\title{
The 'ins' and 'outs' of integrin signalling
}

Integrins can transmit signals from the inside of the cell to the outside (inside-out signalling) and vice versa (outside-in signalling). Integrin signalling involves the binding of proteins such as talin and $G \alpha_{13}$ to the cytoplasmic domain of $\beta$ integrins, but how such protein interactions determine the direction of signalling is unclear. Shen et al. show that talin and $\mathrm{Ga}_{13}$ coordinate a directional switch in integrin signalling by binding to integrin $\beta 3$ in a mutually exclusive manner, at discrete times.

Stimulators of $G$ protein-coupled receptors, such as thrombin, activate inside-out signalling by inducing talin binding to allb $\beta 3$ in platelets, triggering cell adhesion and aggregation. Platelet spreading, full-scale aggregation and thrombosis (blood clot formation) requires outside-in signalling through the same integrin. Intriguingly, the authors found that talin and $\mathrm{Ga}_{13}$ bind $\beta 3$ in a mutually exclusive but temporally regulated manner, in opposing 'waves'. A first wave of talin association with $\beta 3$ occurred when inside-out signalling was stimulated in platelets by thrombin, but prior to the onset of integrin-mediated platelet aggregation. Talin- $\alpha$ llb $\beta 3$ interactions were diminished once outside-in signalling was initiated. A second wave of talin binding to $\beta 3$ occurred after full platelet aggregation and correlated with shrinking blood clots. The binding of $\mathrm{Ga} \alpha_{13}$ to $\beta 3$ peaked between the two waves of talin binding, and is thus likely to promote outside-in signalling.

Further experiments confirmed that the waves of $\beta 3$-talin and $\beta 3-\mathrm{Ga}_{13}$ interactions mediate the switch in direction of integrin signalling. Talin-depleted platelets had aggregation, adhesion and spreading defects, which were rescued by chemicals that induce integrin activation independently of inside-out signalling. As cell spreading requires early outside-in signalling, these data confirm that talin mediates inside-out but not early outside-in signalling.

The authors had observed that an EXE motif in $\beta 3$ is required for $\mathrm{G} \alpha_{13}$ binding but not talin binding. Thus, they expressed wild-type $\beta 3$, or $\beta 3$ in which the EXE motif was mutated, in $\beta 3$-null bone marrow stem cells and transplanted

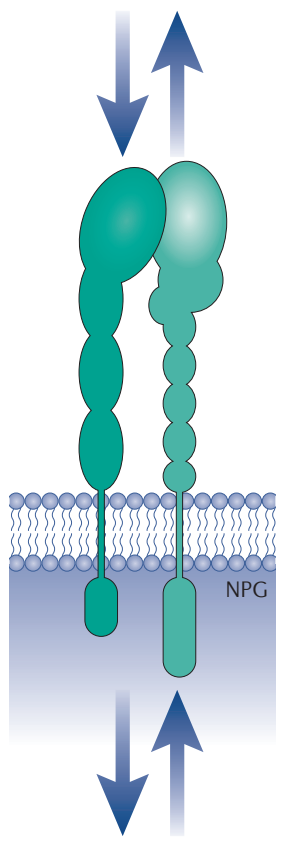

them into irradiated $\beta 3$-null mice. No $\beta 3-G \alpha_{13}$ interaction was observed in platelets from mice expressing $\beta 3$ with the mutated EXE motif, which were also defective in cell spreading although $\beta 3$-talin interactions were unaffected. Thus, the binding of $\mathrm{Ga}_{13}$ to the EXE motif is essential for outside-in signalling but not inside-out signalling.

Finally, as outside-in signalling is important for full-scale thrombi that clog blood vessels, and inside-out signalling triggers initial platelet adhesion and thrombus formation at wounds to prevent bleeding, the authors attempted to design anti-thrombotic molecules against the $\beta 3$ EXE motif. They synthesized several myristoylated EXE motif-containing $\beta 3$ peptides, one of which specifically inhibited early outside-in signalling. This inhibitor was as potent as the commonly used integrin antagonist eptifibatide (Integrilin; Millennium Pharmaceuticals) in inhibiting thrombosis in mice, and, unlike eptifibatide, it could prevent thrombosis without causing bleeding.

So, this study reveals how integrin signalling switches direction and makes use of this mechanistic information to design a novel anti-thrombotic drug. Katharine H. Wrighton switch in integrin signalling

ORIGINAL RESEARCH PAPER Shen, B. et al. A directional switch of integrin signalling and a new anti-thrombotic strategy. Nature http://dx.doi. org/10.1038/nature12613 (2013) 